\title{
Apport de la modélisation hydro-sédimentaire 1D à l'étude d'une retenue de forme allongée : application à la retenue de Génissiat sur le Haut-Rhône français
}

\author{
Lucie GUERTAULT ${ }^{1}$, Benoît CAMENEN², André PAQUIER ${ }^{2}$, \\ Jean-Baptiste FAURE2 ${ }^{2}$ Christophe PETEUIL ${ }^{3}$
}

\author{
1. Department of Biological and Agricultural Engineering, North Carolina State University, Campus Box 7625, Raleigh, NC 27695, Etats-Unis - \\ e-mail : lsguerta@ncsu.edu \\ 2. Irstea, UR RiverLy, centre de Lyon Villeurbanne, 5 rue de la Doua, CS 2024469625 Villeurbanne, France - e-mail : benoit.camenen@irstea.fr \\ andre.paquier@irstea.fr, jean-baptiste.faure@irstea.fr \\ 3. Compagnie Nationale du Rhone, 2 rue Andre Bonin, 69316 Lyon Cedex 04, France - e-mail : c.peteuil@cnr.tm.fr
}

\begin{abstract}
RÉSUMÉ. - L'étude de la dynamique sédimentaire dans les retenues de barrage reste complexe du fait de la combinaison entre les événements naturels et la gestion du barrage. Dans ce papier, nous montrons l'intérêt de la modélisation numérique pour compléter les études morphologiques basées sur l'analyse de mesures. Dans le cas d'une retenue de forme allongée, cette modélisation peut être réalisée avec des codes numériques unidimensionnels (1D). Les codes 1D RubarBE et AdisTS, développés à Irstea Lyon, ont été appliqués sur la retenue de Génissiat sur le Haut-Rhône français. Ils ont permis de calculer des paramètres hydrauliques utiles au découpage du site d'étude en tronçons homogènes. De plus, les résultats de simulations ont aidé à mettre en évidence la dynamique sédimentaire des trois zones décrivant la retenue, i.e. le tronçon fluvial dominé par le charriage, la retenue amont par la suspension graduée de sables et la retenue aval par la suspension d'argile et de limons. Enfin, la modélisation numérique est utile pour la réalisation de scénarios prédictifs et le test de modes de gestion alternative.
\end{abstract}

Mots-clés : transport solide, morphodynamique, retenue de barrage, modélisation 1D

\section{Interest of 1D modelling to study elongated dam reservoir: application to the Génissiat reservoir on the French Upper Rhône}

\begin{abstract}
Studying sediment dynamics in dam reservoirs remains an issue because processes are influenced by both natural events and dam management. In this paper, we showed the interest of applying numerical models to complete geomorphological studies based on the analysis of field measurements. In case of an elongated reservoir, one-dimensional (1D) modelling can be sufficient to complete this analysis. One-dimensional codes RubarBE and AdisTS, developed at Irstea Lyon, have been applied to the Génissiat reservoir located on the French Upper Rhône River. These codes enable computing hydraulic parameters useful for the reservoir delineation into homogeneous reaches. Moreover, simulation results showed the specific sediment dynamics of the three main zones describing the reservoir : bedload is prevailing in the free flow segment, graded sand suspension in the upper part of the reservoir, and fine sediment (clay, silt) suspension in the lower one. Eventually, an important interest of the numerical modelling is that predictive scenarii and alternative dam management can be tested easily.
\end{abstract}

Keywords: sediment transport, morphodynamics, dam reservoir, 1D modelling

\section{INTRODUCTION}

Les barrages sont construits en travers des cours d'eau pour permettre le stockage de l'eau à des fins de contrôle des crues, d'irrigation, d'alimentation en eau potable ou de production hydroélectrique. Ils forment des obstacles aux échanges longitudinaux dans les cours d'eau, et les retenues créées en amont sont des éléments du système fluvial à part entière qui affectent sensiblement le transit sédimentaire. Les retenues de barrage sont ainsi caractérisées par une augmentation artificielle de la profondeur d'eau de l'amont vers l'aval, impliquant une réduction des contraintes hydrauliques et un important tri granulométrique. On retrouve ainsi à l'amont de la retenue des sédiments grossiers (sables et graviers) formant un delta se propageant vers l'aval alors que les sédiments fins (argiles, limons) se déposent généralement dans les zones les plus profondes (à proximité du barrage) par couches horizontales. L'accumulation au fil du temps des sédiments dans une retenue est inéluctable jusqu'à ce qu'elle ait atteint un nouvel état d'équilibre. De fait, les barrages sont conçus avec des durées de vie finies. Un enjeu important pour les gestionnaires est donc de gérer au mieux leur ouvrage afin de limiter les risques associés au comblement des retenues et assurer leur longévité. Pour cela, différentes solutions permettant de favoriser le transit des sédiments vers l'aval peuvent être mises en place, comme les chasses hydrauliques, tunnels de dérivation « by-pass », dragages, etc. [Peteuil et al., 2013]. Le coût global de ces opérations peut cependant être très important et leur efficacité pourrait parfois être sensiblement optimisée. Dans cette perspective, 
l'outil numérique, une fois validé, peut permettre de mieux comprendre la dynamique sédimentaire d'une retenue, et présente l'avantage de pouvoir tester et comparer une multitude de scénarios de gestion alternatifs. Il constitue un outil d'aide à la décision précieux qui complète judicieusement les connaissances acquises par retour d'expériences.

Pour des raisons évidentes de coût de construction, de nombreuses retenues de barrage ont été créées dans des vallées étroites et profondes. Elles présentent une forme allongée et sont caractérisées par une dimension longitudinale prépondérante par rapport à la dimension transversale, permettant l'utilisation d'un modèle 1D. L'objet de ce travail est de proposer une méthodologie d'étude d'une retenue de forme allongée à l'aide d'une modélisation unidimensionnelle (1D) et de montrer les apports de cette modélisation pour l'étude de la dynamique sédimentaire sur des retenues allongées. Ainsi, deux codes de calculs développés à Irstea Lyon (Adis-TS, Guertault et al., [2016] ; RubarBE, El kadi Abderrezzak, K., \& Paquier, A. [2009], Camenen et al.[2017]) sont appliqués à la modélisation sédimentaire d'une retenue allongée soumise à des opérations de chasse régulières : la retenue de Génissiat sur le Haut-Rhône français. Après une rapide description du site d'étude, la retenue de Génissiat, et des données disponibles, une discussion sur les apports de la modélisation unidimensionnelle est proposée, d'abord sur le découpage en tronçons homogènes puis la compréhension de la dynamique sédimentaire.

\section{LA RETENUE DE GÉNISSIAT}

\section{II.1. Localisation}

La retenue de Génissiat est située sur le Haut-Rhône français. Elle est gérée par la Compagnie Nationale du Rhône (CNR) et est exploitée depuis 1948. La Figure 1 donne un aperçu général de la localisation de la retenue et des principaux sites de mesures exploités en continu ou temporairement lors des opérations de gestion sédimentaire. Le barrage de Génissiat est un barrage de type poids d'une hauteur de $104 \mathrm{~m}$, avec une chute maximale de $67 \mathrm{~m}$. La centrale hydro-électrique peut turbiner un débit maximal de $750 \mathrm{~m}^{3} / \mathrm{s}$. L'exploitation de la retenue présente un cycle journalier et hebdomadaire, avec une production en journée et le remplissage de la retenue pendant la nuit en conservant un débit réservé de $18 \mathrm{~m}^{3} / \mathrm{s}\left(5 \mathrm{~m}^{3} / \mathrm{s}\right.$ avant 2013). Le barrage de Génissiat comprend différents orifices placés dans une configuration tridimensionnelle :

- Une vanne de fond localisée en rive droite environ $200 \mathrm{~m}$ à l'amont du barrage. Son radier est situé à la cote 262,6 m, qui constitue le point bas de la retenue ;

- Une vanne de demi-fond localisée en rive gauche environ $50 \mathrm{~m}$ en amont du barrage. Son radier est situé à la cote $285,9 \mathrm{~m}$;

- Un évacuateur de surface, localisé dans le parement du barrage en rive droite. Son radier est à la cote $316,80 \mathrm{~m}$.

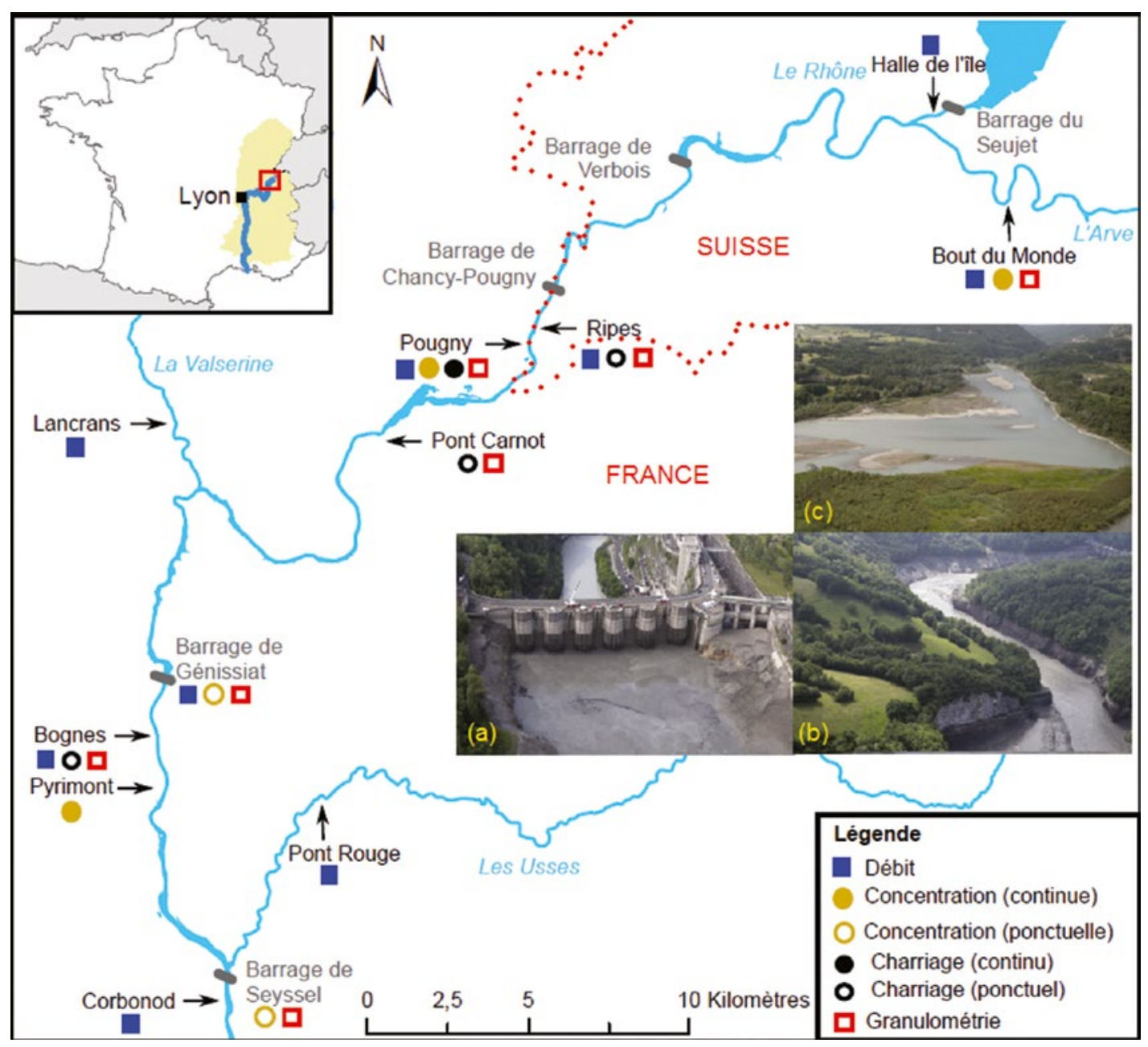

Figure 1 : Localisation de la retenue de Génissiat sur le Haut-Rhône français et des différentes stations de mesures hydrosédimentaires. (a) Barrage de Génissiat, (b) gorges en amont du barrage, (c) zone de l'Étournel en amont du pont Carno). 
La retenue s'étend sur $23 \mathrm{~km}$ du barrage de Génissiat à la zone de l'Etournel en amont du pont Carnot. Elle présentait initialement un volume de 56 millions de $\mathrm{m}^{3}$ dont 12 millions de $\mathrm{m}^{3}$ utilisable pour la production hydroélectrique ; ce volume est aujourd'hui d'environ 40 millions de $\mathrm{m}^{3}$ [Guertault et al., 2014]. A Pougny, la retenue de Génissiat reçoit les eaux d'un bassin versant de $10320 \mathrm{~km}^{2}$. Le régime du Haut-Rhône est nivo-pluvial avec un module inter-annuel de $355 \mathrm{~m}^{3} / \mathrm{s}$. Les débits instantanés des crue décennale et centennale sont respectivement estimés à 1300 et $1500 \mathrm{~m}^{3} / \mathrm{s}$ à Pougny.

\section{II.2. Évolutions bathymétriques}

La retenue est composée de deux unités géographiques distinctes, séparées par le Pont Carnot (Figure 1). A l'amont du Pont Carnot se situe la zone alluviale de l'Étournel. De nombreuses îles et bancs, parfois immergés y sont formés. Le Pont Carnot, situé au niveau d'un seuil rocheux et d'une courbe importante marque l'entrée dans un canyon étroit découpé dans le calcaire. Les trois quarts de la retenue sont situés dans ces gorges. Les évolutions bathymétriques depuis la mise en eau du barrage et la granulométrie de fond sont très liées à la géométrie de la retenue (Figure 2). Il est à noter que les évolutions du thalweg sont généralement représentatives de l'évolution morphologique du lit [Guertault et al., 2014] et cette valeur est souvent la seule disponible pour les données anciennes. On distingue ainsi trois zones :

- Un tronçon fluvial peu influencé par le barrage, situé en amont du pont Carnot. Il est sujet à des évolutions bathymétriques très faibles et présente un lit pavé composé de galets $\left(d_{50} \approx 100 \mathrm{~mm}\right)$.

- Une retenue amont incluant les tronçons de l'Écluse, Léaz et Grésin assez dynamique avec une tendance générale légèrement en exhaussement et un lit sableux ;

- Une retenue aval (tronçon de Bellegarde et canyon d'Arlod) caractérisée par des dépôts très importants principalement composés d'argile et limon.

Historiquement, le comblement de la retenue de Génissiat est intervenu majoritairement à l'occasion des opérations de chasses organisées à l'initiative des exploitants suisses.

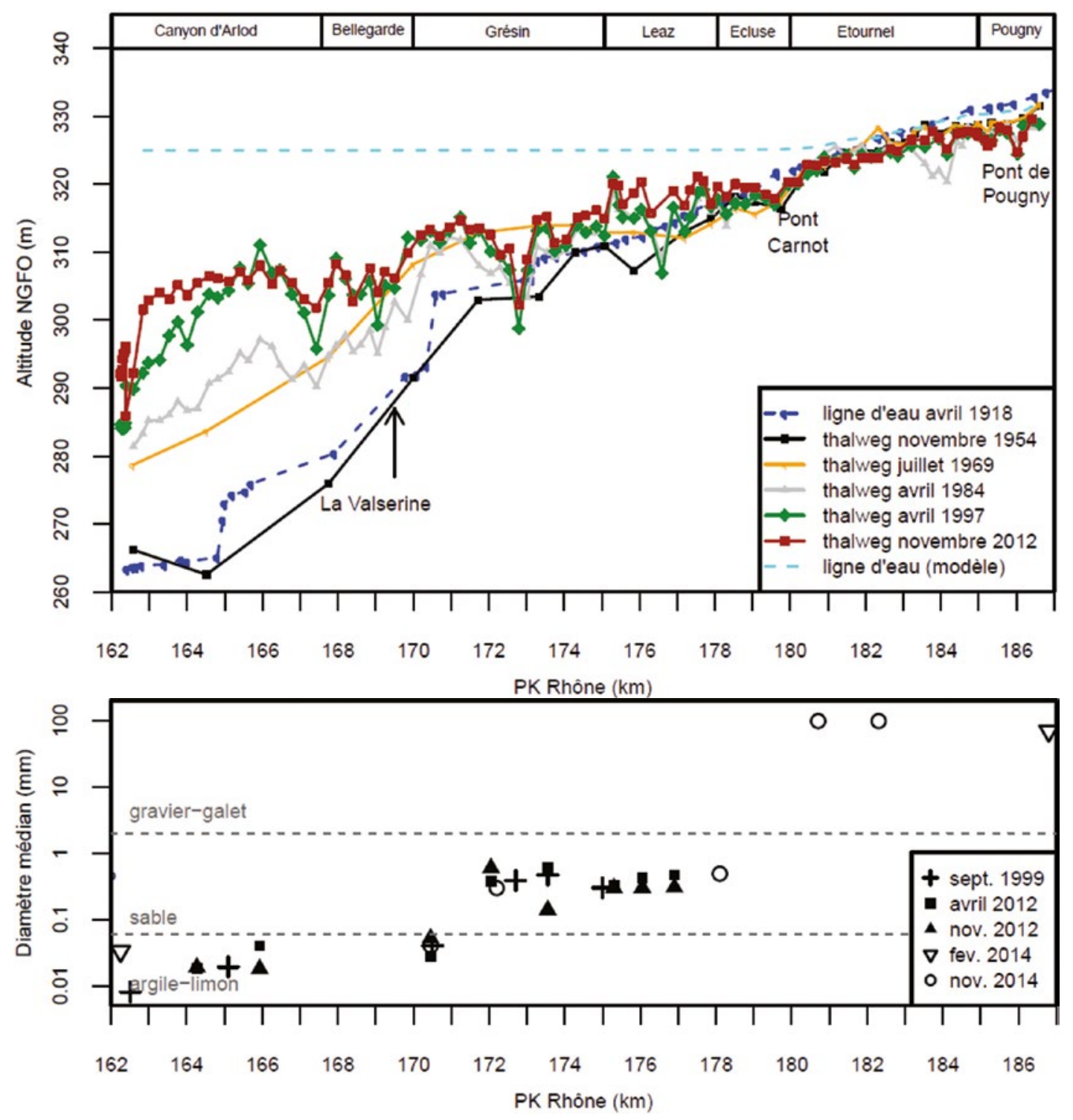

Figure 2 : Retenue de Génissiat: (a) Évolutions bathymétriques de la retenue et (b) Répartition spatiale de la granulométrie récente. 
L'objectif de ces opérations a été de prévenir d'éventuels risques de sur-inondation dus à la sédimentation dans les retenues situées au droit et en aval de la ville de Genève (Verbois et Chancy-Pougny respectivement). La régulation de ces apports solides dans la retenue de Génissiat a permis de préserver efficacement la qualité écologique du Haut-Rhône depuis 1948. Cette régulation a été rendue possible par la mise en place d'un protocole de gestion original du plan d'eau et des vannes du barrage [Peteuil et al., 2013] mais elle s'est faite aussi au prix d'une sédimentation significative en amont du barrage.

En période d'exploitation normale, la retenue de Génissiat est gérée à cote haute. Bien que les conditions hydrauliques dans la retenue soient potentiellement favorables au dépôt, la sédimentation est beaucoup plus marginale durant ces périodes car les apports sédimentaires sont réduits par la présence des barrages amont.

\section{APPORT DE LA MODÉLISATION UNIDIMENSIONNELLE}

\section{III.1. Codes de calculs utilisés}

Deux codes de calcul développés à Irstea Lyon ont été utilisés pour cette étude. Le premier, Adis-TS [Guertault et al., 2016], résout l'équation d'advection-dispersion avec une prise en compte des termes sources (érosion et dépôt de sédiments) mais sans évolution effective des fonds. Du fait du couplage faible avec le module hydraulique (MAGE) et de la parallélisation du code de calcul, les simulations peuvent être aisément réalisées sur le long terme (plusieurs décennies) avec une prise en compte de plusieurs classes de sédiments et polluants. Une première application sur la sédimentation d'une lône avait donné des résultats encourageants [Camenen et al., 2013]. Le modèle est ici validé sur la retenue de Génissiat en se basant sur une étude préliminaire des flux transitant sur la retenue et des évolutions bathymétriques de cette dernière [Guertault et al., 2014]. Il est à noter de plus qu'un module spécifique à la retenue de Génissiat a été mis en place pour mieux prendre en compte le gradient vertical des concentrations au droit des orifices étagés le long du parement du barrage [Guertault et al., 2016].

Le second code de calcul, RubarBE [El kadi Abderrezzak, K., \& Paquier, A., 2009 ; Camenen et al., 2017] résout l'équation de conservation de la masse (ou l'équation d'Exner) qui traduit l'évolution du fond suite au transport solide. Le débit solide est estimé à partir d'une loi de capacité de transport via une équation de chargement qui permet de simuler le transport sédimentaire en dehors de l'équilibre. Ce modèle a été validé pour des écoulements complexes comme les ruptures de barrage [El kadi Abderrezzak, et al., 2008] et considère donc un transport solide par charriage (et suspension graduée) prépondérant. Le code a la particularité de prendre en compte un mélange de sédiment non par un système multi-classes mais par l'intermédiaire de deux paramètres seulement (diamètre médian et étendue granulométrique) associés à des lois d'échange adaptées [Camenen et al., 2017].

Les modules hydrauliques ont été calés sur plusieurs lignes d'eau mesurées sur la retenue à différents débits en prenant soin de tenir de la variabilité spatiale de la granulométrie [Guertault, 2015].

\section{III.2. Modélisation des flux de Matière En Suspension (MES)}

Le modèle Adis-TS a permis de reproduire correctement les masses de sédiments fins et de sables transportées au cours d'épisodes de chasse ou de périodes d'inter-chasse après un calage simple du terme de concentration d'équilibre et en décomposant la courbe granulométrique des MES en six classes, trois pour les argiles et limons, trois pour les sables [Guertault et al., 2016]. Le module complémentaire spécifique à la zone du barrage a permis de prendre en compte l'effet des fortes profondeurs sur la distribution verticale des sédiments et donc de mieux décrire qualitativement et quantitativement les sédiments transitant dans les différents ouvrages du barrage (vanne de fond, vanne de demi-fond, évacuateur de surface, Figure 3). La modélisation multi-classes du transport des sédiments fins et des sables s'est avérée particulièrement adaptée pour reproduire la dynamique des différentes fractions (présence de sables dans la vanne de fond mais pas dans la vanne de demi-fond, Figure 3 ) et les flux tout au long du système étudié. Les écarts observés peuvent principalement être attribués à des érosions locales mal reproduites (effondrement de berge, effets bidimensionnels, etc.) ou à une évaluation des sédiments disponibles (conditions initiales) incorrecte.

Enfin, le modèle Adis-TS montre des capacités intéressantes pour la simulation de scénarios prédictifs. Il a ainsi pu être utilisé pour simuler et départager les différents protocoles envisagés pour les chasses du Haut-Rhône du mois de mai 2016. Le modèle a aussi permis d'étudier un protocole de gestion alternatif au cours d'une crue [Guertault, 2015].
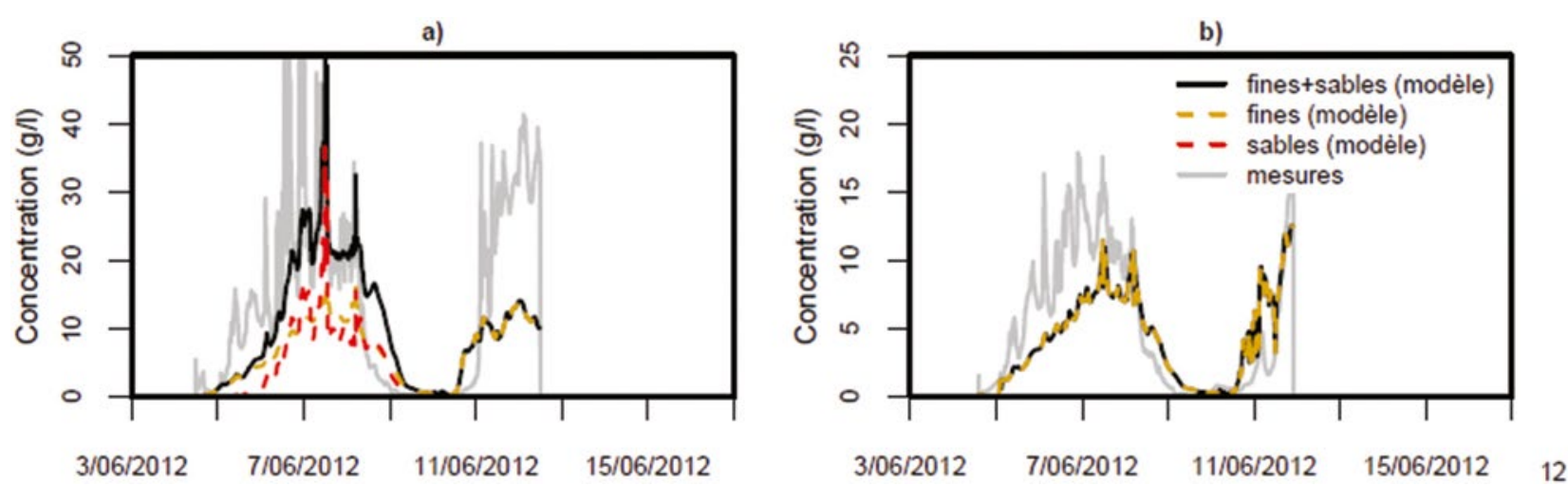

Figure 3 : Comparaison entre les concentrations mesurées et modélisées au barrage de Génissiat lors de la chasse de 2012 ( $a$ : vanne de fond ; $b:$ vanne de demi-fond). 


\section{III.3. Découpage en tronçons homogènes}

Une classification consiste à découper un cours d'eau en entités homogènes. Elle est généralement utilisée pour l'établissement de diagnostics géomorphologiques. La définition d'entités homogènes s'effectue par détection de discontinuités longitudinales locales de variables hydro-morphologiques caractéristiques (largeur active, pente, etc.). Classiquement visuelle cette détection peut être effectuée par des méthodes statistiques [Levandier et al., 2012]. Afin d'obtenir un découpage pour une retenue de barrage qui mette en évidence l'importance et l'activité des processus, une nouvelle méthode a été proposée en s'appuyant notamment sur la modélisation hydraulique 1D [Latapie et al., 2014]. La modélisation permet en effet de calculer des paramètres hydrauliques difficilement calculables par des méthodes classiques dans le cas de systèmes anthropisés.

La méthode proposée s'appuie ainsi sur ces trois familles de paramètres :

— le style fluvial, correspondant à un découpage classique (tracé en plan, profil longitudinal, sinuosité, largeur) ;

- des paramètres hydrodynamiques (ratio largeur sur hauteur, contraintes de cisaillement au fond), estimés pour des scénarios hydrauliques représentatifs des écoulements dans la retenue en chasse (niveau bas) et en période d'interchasses (niveau haut) ;

- les évolutions morphologiques consécutives aux épisodes de chasse et d'inter-chasses.

Le découpage a permis de retenir 20 tronçons homogènes sur l'ensemble de la retenue [Guertault et al., 2017]. Ce découpage sera utilisé par la suite pour comparer les résultats de simulation aux mesures in situ (Figure 4).

\section{III.4. Modélisation des évolutions bathymétriques}

Les intérêts de la modélisation hydro-sédimentaire sont de mettre en évidence les processus hydro-sédimentaires dominants dans la retenue, d'y reproduire les évolutions morphologiques de la retenue et les flux sédimentaires transitant par le barrage, au cours d'épisodes de chasse et de périodes d'inter-chasses. Les deux codes AdisTS et RubarBE ont été utilisés pour modéliser quelques événements pour lesquels suffisamment de données de calage sont disponibles. Ces données portent en particulier sur les évolutions bathymétriques de la retenue, les variations spatiales et temporelles des flux sédimentaires ou encore la composition granulométrique des matériaux déposés et en transit. L'importance de la collecte de ces données doit être rappelée ici car bien que chronophage et coûteuse, elle est tout à fait prépondérante pour comprendre a posteriori le fonctionnement de ces systèmes complexes.

La Figure 4 présente une comparaison entre les bilans volumiques mesuré et simulé dans la retenue de Génissiat suite à la chasse de 2003. Le code AdisTS a permis de reproduire le dépôt sélectif des sédiments dans la retenue et le tri granulométrique observé [Guertault, 2015]. Les résultats sur l'évolution morphologique de la retenue sont corrects pour les deux tiers aval de la retenue où les sédiments fins (argiles, limons et sables) prédominent (Figure $4 \mathrm{~b}$ ). Ce constat suggère que la suspension est le mode de transport principal sur les tronçons 10 à 20 . Nous observons néanmoins une limite concernant les sables car leur propagation semble être surestimée. De plus, le modèle ne reproduit pas toujours parfaitement l'évolution des fonds, ce qui pourrait être lié au fait que la géométrie des fonds n'est pas mise à jour dans le modèle. À l'inverse, le modèle RubarBE
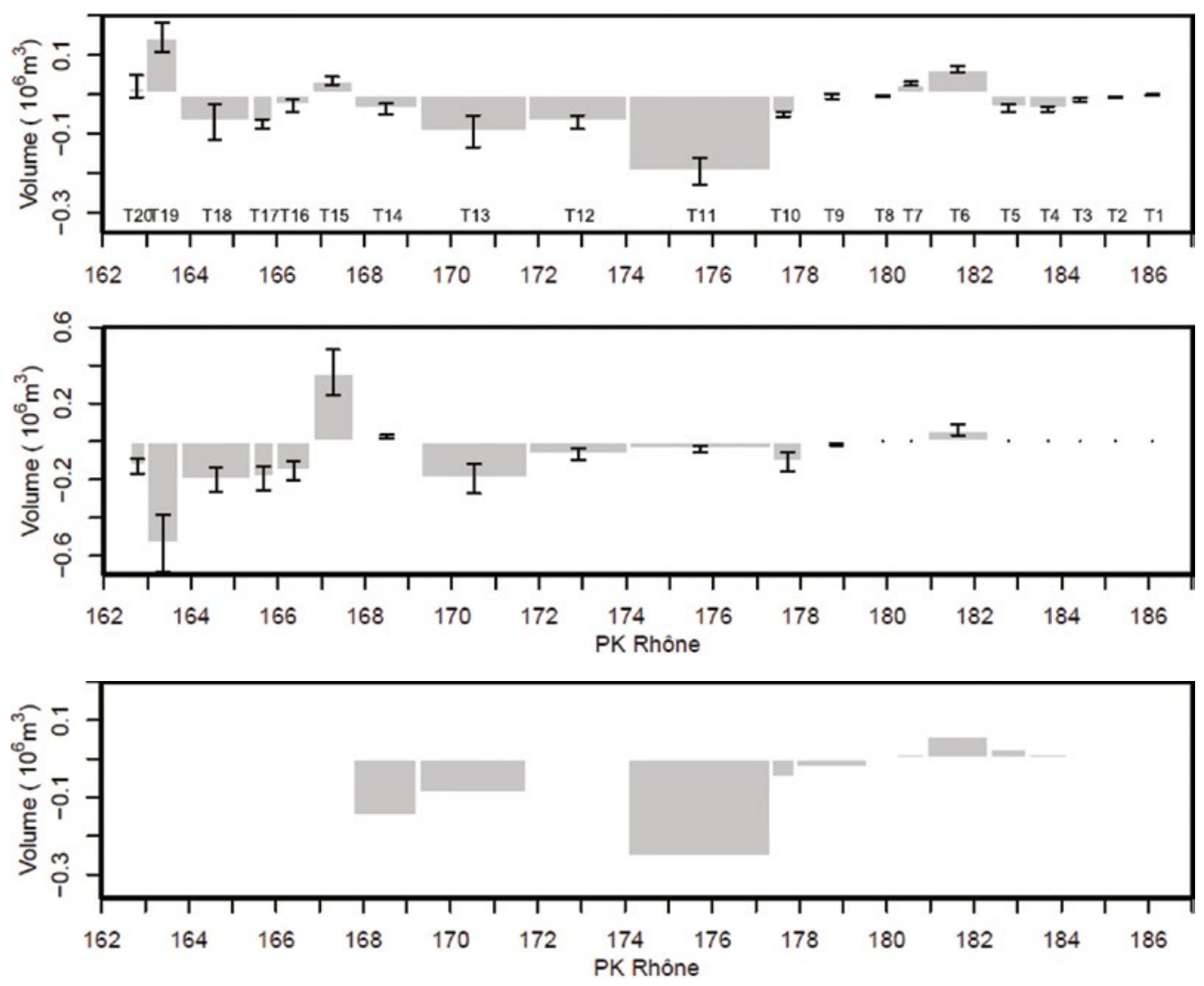

Figure 4 : Bilan volumique dans la retenue de Génissiat suite à la chasse de 2003 : (a) mesuré et (b) simulé à l'aide du code AdisTS et (c) simulé à l'aide du code RubarBE. 
(appliqué avec la formule de van Rijn [1984] incluant le charriage et/ou la suspension graduée) donne de bons résultats sur les deux tiers amont de la retenue où les sédiments plus grossiers (sables, graviers, galets) prédominent. $\mathrm{Ce}$ constat suggère que la charge de fond prédomine sur les tronçons 1 à 13. En particulier, sur les tronçons 9 à 13, la suspension graduée de sables semble prédominer sur le charriage. En effet, l'application de la modélisation numérique avec la formule de transport par charriage seule (terme de suspension dans la formule de Van Rijn écarté) sous-estime largement les évolutions. La partie amont reste cependant difficile à modéliser du fait d'un transport principal de sédiments sableux et graveleux sur un lit pavé de galets et d'une estimation difficile et incertaine des apports amont.

La modélisation numérique confirme bien une dynamique de transport spécifique à chacune des trois zones identifiées : - Un transport solide par charriage prédominant sur le tronçon fluvial ;

- Un transport solide par suspension graduée (charge de fond) prédominant sur la retenue amont ;

- Un transport solide par suspension de fines sur la retenue aval.

\section{CONCLUSIONS}

Cet article présente plusieurs intérêts de la modélisation numérique pour l'étude d'une retenue de forme allongée. La modélisation hydro-sédimentaire de la retenue de Génissiat a montré qu'une approche unidimensionnelle était suffisante pour modéliser les processus dans la majeure partie de la retenue. Pour les tronçons présentant une géométrie complexe (amont immédiat du barrage avec de grandes profondeurs et plusieurs ouvrages, zone de l'Étournel avec des chenaux multiples), des modèles 2D peuvent être développés localement afin d'améliorer la compréhension des processus mais restent difficiles à valider [Duron, 2014].

Une fois calé, un modèle numérique hydraulique permet l'obtention de paramètres hydrauliques (ratio largeur sur hauteur, contraintes de cisaillement au fond) qui sont complémentaires des paramètres utilisés classiquement en géomorphologie. Ils s'avèrent d'une grande utilité pour le découpage en tronçons homogènes de la zone d'étude et la caractérisation de chacune de ces entités. Cette approche apporte une réelle plus-value dans le cas de sites fortement anthropisés où les méthodes classiques trouvent rapidement leurs limites (débit de plein bord, etc.).

La modélisation numérique complète les jeux de mesures acquis (flux, évolution morphologique) pour la compréhension des phénomènes physiques. Ainsi, les codes RubarBE et AdisTS ont permis de confirmer la dynamique sédimentaire prépondérante sur les trois zones décrivant la retenue de Génissiat, soit un charriage prépondérant sur le tronçon fluvial, une suspension graduée des sables sur la retenue amont et une suspension de fines (argiles et limons) sur la retenue aval. Le code AdisTS a permis de reproduire le dépôt sélectif des sédiments lié à la diminution des contraintes ainsi que les flux sortants. Cependant, une difficulté reste la modélisation des sables pouvant se propager par charriage et suspension. Les deux modèles montrent que les sédiments sableux peuvent atteindre le barrage mais l'estimation de leur vitesse de propagation reste encore incertaine.

Enfin, différents scénarios prédictifs peuvent être simulés à partir des modèles numériques et peuvent permettre, en complément des connaissances expertes des gestionnaires, une quantification des différents scénarios de gestion et une inter-comparaison plus aisée de leurs impacts. Ainsi, le modèle AdisTS a été utilisé par CNR pour préparer l'opération d'accompagnement des chasses des barrages suisses du Haut-Rhône de mai 2016 [Guertault, 2015].

\section{RÉFÉRENCES}

Camenen B., Andries E., Faure J.-B., De Linares M., Gandilhon F. \& RACCASI G. (2013) - Experimental and numerical study of long term sedimentation in a secondary channel : example of the Beurre island on the Rhône River, France. Proc. 12th Int. Symp. on River Sedimentation, 2-5 sept. 2013, Kyoto, Japon, CD-ROM, 8 p.

Camenen B., Beraud C., Le Coz J. \& Paquier A. (2017) - 1D numerical simulation of sediment downstream fining during bed aggradation using a simplified grain size description. Journal of Hydraulic Research (sous presse).

Duron L. (2014) - Modélisation bidimensionnelle et tridimensionnelle des écoulements à l'amont du barrage de Génissiat. Mémoire de Master, Grenoble INP ENSE3, 60 p.

El Kadi Abderrezzak K., Paquier A. \& Gay B. (2008) One-dimensional numerical modelling of dam-break waves over movable beds : application to experimental and field cases. Environmental Fluid Mechanics, 8 : 169-198.

El Kadi AbderrezzaK K., \& Paquier A. (2009) - One-dimensional numerical modeling of sediment transport and bed deformation in open channels. Water Resources Research, 45 (W05404) : 1-20.

Guertault L. (2015) - Évaluation des processus hydro-sédimentaires d'une retenue de forme allongée : application à la retenue de Génissiat sur le Haut-Rhône. Thèse de doctorat. Université Claude Bernard, Lyon 1, France, 262 p.

Guertault L., Camenen B., Peteuil C. \& Paquier A. (2014) Long term evolution of a dam reservoir subjected to regular flushing events. Advances in Geosciences, 39 : 89-94.

Guertault L., Camenen B., Peteuil C., \& Paquier A. (2017) Sediment transport dynamics in an elongated dam reservoir subjected to management operations. Earth Surface Processes and Landforms (sous presse).

Guertault L., Camenen B., Peteuil C., Paquier A. \& Faure J.-B. (2016) - One dimensional modelling of suspended sediment dynamics in dam reservoirs. Journal of Hydraulic Engineering, 142(10.1061) : 1-9.

Kondolf G. M. (1997) - Hungry water : effects of dams and gravel mining on river channels. Environmental management, 21(4) : 533-551.

Latapie A., Camenen B., Rodrigues S., Paquier A., Bouchard J.-P. \& Moatar F. (2014) - Classifying anthropogenised river reaches to assess channel changes using hydraulic parameters. Catena, 121 : 1-12.

Leviandier T., Alber A., Le Ber F. Et Piegay H. (2012) Comparison of statistical algorithms for detecting homogeneous river reaches along a longitudinal continuum. Geomorphology, 138(1) : 130-144.

Peteuil C., Fruchart F., Abadie F., Reynaud S., Camenen B., And Guertault L. (2013) - Sustainable management of sediment fluxes in reservoir by environment friendly flushing : the case study of the Génissiat dam on the Upper-Rhône River (France). Proc. of the 12th Int. Symp. on River Sedimentation (ISRS2013), 2-5 sept. 2013, Kyoto, Japon, CD-ROM, 8 p.

Thornton K. W., Kennedy R. H., Carroll J. H., Walker W. W., Gunkel R. C. Eт Ashby S. (1981) - Reservoir sedimentation and water quality : an heuristic model. Proc. of the symposium on surface water impoundments, 2-5 Juin 1980, Minneapolis, Minesota, États-Unis, vol. 1, pages 654-661. ASCE.

VAN RIJN L. C. (1984) - Sediment transport, part I : bed load transport / part II : suspended load transport. Journal of Hydraulic Engineering, 110(10) : 1431-1456/1613-1641. 